\title{
Impacts of Coal Railroad Transportation Project on GDP Promotion and Unemployment Reduction in Bengkulu Province, Indonesia
}

\author{
PAULUS KURNIAWAN \\ Doctorate Candidate from Faculty of Economics at Postgraduate Program, University of Udayana, \\ Denpasar, Indonesia \\ Email: pikeke.kho@gmail.com \\ KEMBAR SRI BUDHI
}

Professor, Faculty of Economics at Postgraduate Program, University of Udayana, Denpasar, Indonesia SUYANA UTAMA

Professor, Faculty of Economics at Postgraduate Program, University of Udayana, Denpasar, Indonesia

\section{MAHAENDRA YASA}

Doctor, Faculty of Economics at Postgraduate Program, University of Udayana, Denpasar, Indonesia

\begin{abstract}
A massive, advanced and integrated railroad system for coal transportation (referred to hereafter as "Project") from Muara Enim, Sumatra Selatan Province to the new coal port at Pulau Baai, Bengkulu Province, Indonesia is developed by a private investor together with the local government (PEMDA) of Bengkulu Province to make the best use of abundant coal resources in the region. This paper analyzes the impact of this Project on the Bengkulu economy, which is currently considered low. The Gross Domestic Product (GDP) is adopted as the economic indicator. The study combines the theories of export base and economic base, economic and regional developments, cost-benefit analysis and economic impact study with the empirical data. The results show the Project's financial feasibility with Cost-Benefit Ratio of 1.61, Internal Rate of Return of $21.1 \%$ and Payback Period of 5 years, which will provide a significant contribution to the Bengkulu GDP growth and a decrease of 821,600 people among the unemployed.
\end{abstract}

Keywords: railway coal transportation, GDP, decreasing unemployment, Bengkulu Province, Indonesia. 


\section{INTRODUCTION}

Indonesia has one of the largest coal reserves in the Asia-Pacific region, with proven reserves of 7 billion tons, and these are found mostly in Kalimantan and Sumatra, with resources estimated at 32.9 and 27.3 billion tons respectively (Indonesian Ministry of Energy and Mineral Resource). Sumatra has 27.4 billion tons of coal resources and 2.7 billion tons of proven reserves, according to the Indonesian Coal Mining Association, suitable for power-generating activities, with subbituminous, low sulphur and ash content and average per Kgcaloric value of 5,000 6,000 calories.

Although resources in Sumatra are comparable in quantity with Kalimantan, Sumatra (especially Bengkulu and South Sumatra Provinces) is producing and exporting small quantities of coal compared with its potentials due to inadequate infrastructure (hauling access and port) for coal transportation. To meet this infrastructural inefficiency, a private investor together with the local government (PEMDA) of Bengkulu Province has developed a massive, advanced and integrated coal transportation system ("Project") including the followings:

A double track railway of $\pm 265 \mathrm{~km}$ in length to exclusively transport coal from major coal mines in Muara Enim, South Sumatra Province and around Bengkulu Province, as well as significant coal resources estimated at 16.6 billion metric tons in the immediate hinterland of the railway (Kurniawan, 2010) to a new coal port in Pulau Baai, Bengkulu Province, Sumatra, Indonesia. The Project will have a minimum capacity of 40 millions metric ton per annum (MTPA) and 100 MTPA for future plan.

The introduction of the Project may have significant and positive impacts on Bengkulu economy. According to Bengkulu Statistics Center (Badan Pusat Statistik / BPS Bengkulu, 2010), Bengkulu Province has a low GDP, a low income per capita, and a high rate of unemployment compared with other provinces. Although rich in natural resources, especially coal, Bengkulu has a poor infrastructure and geographical disadvantages of being isolated due to difficult natural terrain along with the neighboring provinces of South Sumatra, Padang and Jambi, which might somehow badly affect its economy.

This study is therefore conducted to analyze the impact of the development of a coal port and railroads to Bengkulu economy. The methodology used in this study is shown 
in Figure 1. The economic indicator is the GDP. The impact on Bengkulu GDP may thus indicate the importance and value of the Project to the economy of Bengkulu.

\begin{tabular}{|l|}
\hline \multicolumn{2}{|c|}{ THEORY } \\
\hline 1. Export Base: \\
Innis (1920), Cramon and Rovayo \\
(2006) \\
2. Economic Base: \\
Richardson (1973) \\
3. Economic Development: \\
Amartya Sen (1999); Stimson, Stough \\
and Robert (2006). \\
4. Regional Development: \\
Anderson (2011); Stimson, Stough and \\
Salazar (2009) \\
5. Cost-Benefit Analysis CBA/COBA): \\
Riley (2006); Sinaga (2009) \\
6. Economic Impact Study: \\
Tom (2010); Pondent (2010); Capozzi \\
(2010); Hall (2010a, 2010b, 2010c) \\
\hline
\end{tabular}

\begin{tabular}{|l|}
\hline \multicolumn{1}{|c|}{ EMPIRICAL DATA } \\
\hline Bengkulu Statistics Center : BPS Bengkulu \\
Province, 2010, 2011. \\
Project Feasibility Study: Kurniawan, 2010. \\
Project Design: Pratama Widya, 2008-2010 \\
Project Cost Estimation: Pratama Widya, 2008- \\
2010. \\
Project Supporting Data: References. \\
Dissertation: Kurniawan, 2012. \\
Research Pustra: Ministry of Indonesian Public \\
Works, 2007 \\
\hline
\end{tabular}

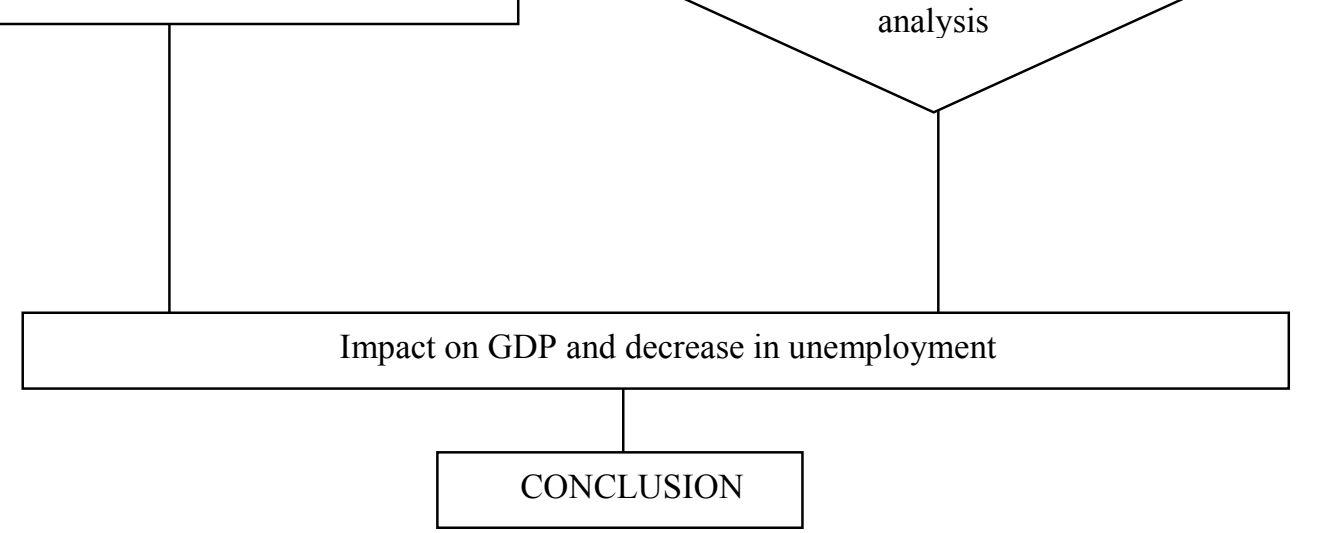

Figure 1: Study Methodology

The export-base theory was introduced by Harold Innis (England), in early 1920, and developed by North (1955), Dusenberry (1950), Andrews (1953) and deeply stressed by Cramon and Rovayo (2006). This theory refers to the Neo-classical 
approach to regional growth based on resource areas in North America with the growth of the industry by exporting goods and services from region to region (Cramon and Rovayo, 2006).

The economic-base theory was first stated by Harry W. Richardson in 1973 (Arsyad, 2010), and it maintains that the main determinants of economic growth in an area are directly related to the demand for goods and services from the outside. Regional development strategies based on this theory usually give emphasis to the national and international markets (Arsyad, 2010).

Economic and regional development theory was well determined by Amartya Sen (1999), [Arsyad (2010); Stimson, Stough and Roberts (2006); Stimson, Stough and Salazar (2009)].

Cost-Benefit Analysis (CBA/COBA) is a technique for assessing the monetary social costs and benefits of an investment project over a given period of time. The investment criteria methods of project and their application might be determined by five models: (1) Net Present Value / Worth (NPV); (2) Cost-Benefit Ratio; (3) Profitability Index; (4) Payback Period; and (5) Internal Rate of Return / IRR (Gray et al., 1986; Riley, 2006; and Sinaga, 2009).

Economic impact study was described and studied by Pondent (2010); Capozzi (2010) and Hall (2010a, 2010b, 2010c).

Econometrical analysis method is carried out in the project's feasibility study on secondary time series data for the years 2011 - 2040, using the regression and path analysis with Eviews program (Kurniawan, 2012).

The framework of variable links is as follows: 


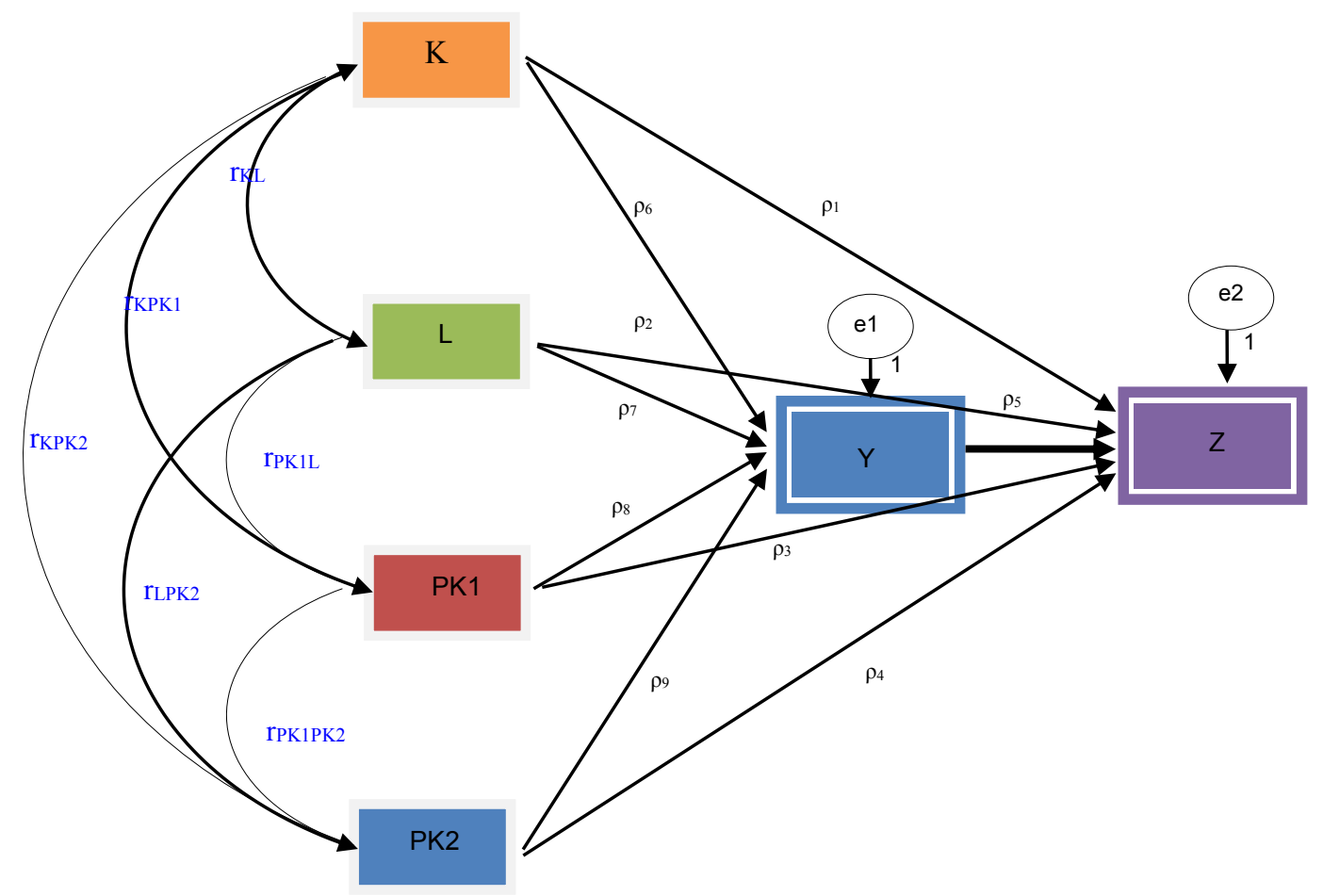

Figure 2: Framework of Variable Links

Independent variables : K, L, PK1, PK2

Dependent variable $\quad: Z$

Intervening variable $: \mathrm{Y}$

Where,

$\mathrm{K}=$ Private investment (Capital)

$\mathrm{L}=$ Labor

PK1 = Government expenditure on development

PK2 = Government expenditure on education and healthcare

$\mathrm{Y}=$ Private output

$\mathrm{Z}=\mathrm{GDP}$

e $=$ Disturbance variable (error)

$r=$ Correlation coefficient

$\rho_{1}, \rho_{9}=$ Path coefficient 


\section{PROJECT DEFINITION STATEMENT}

The project is to develop an integrated coal transportation system from coal mines in Bengkulu and Sumatera Selatan Provinces to Pulau Baai Port in Bengkulu Province. The system is designed to be of high capacity, efficient and reliable, and equipped with advanced facilities to meet the target minimum capacity of 40 MTPA and maximum 100 MTPA in the future. The railroad alignment has been chosen as the most efficient route through the selected areas from the mines to the Pulau Baai Port considering all key factors such as geographical obstacles, existing infrastructure, protected, restricted, populated and environmentally sensitive areas (Kurniawan, 2010).

The coal transportation model is shown in Figure 3, while the adopted key elements in this coal railroad transportation Project are shown in Table 1. The required significant amount of electricity for this Project will be supplied by other parties.
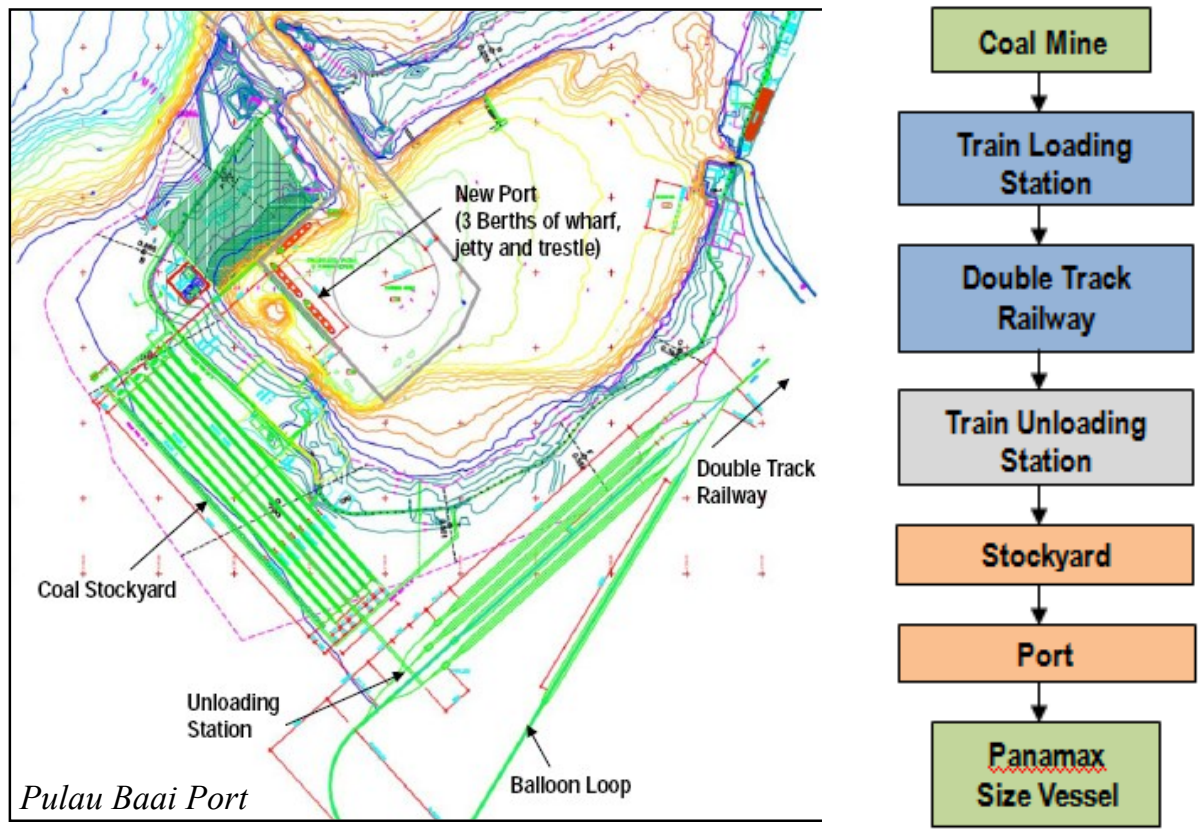

Source: Kurniawan, 2010

Figure 3: Coal Port Plan and Coal Transportation Model 
Table 1: Coal Transportation System - Key Elements

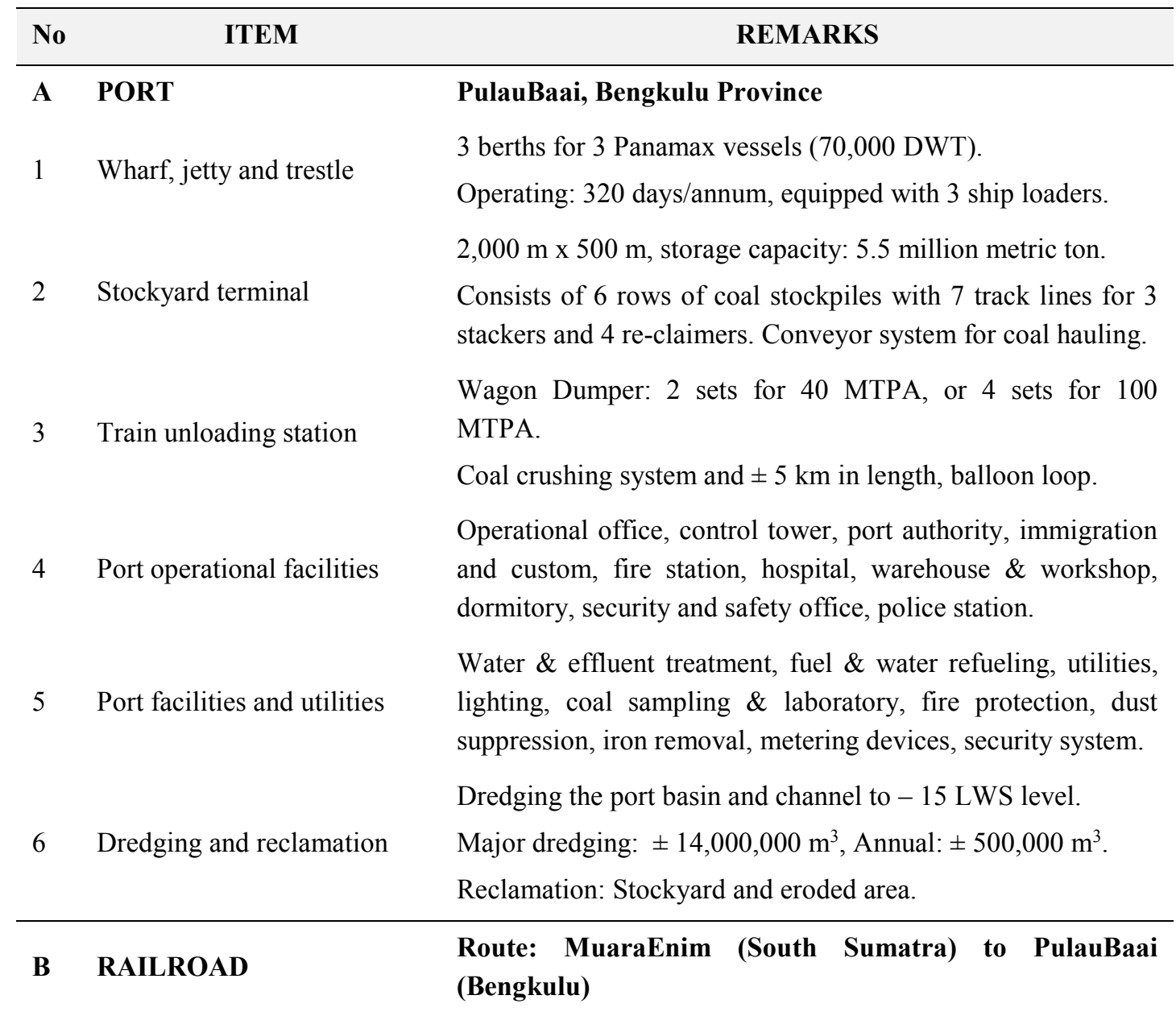

$265 \mathrm{~km}$ double track (50 m ROW), standard gauge 1,435 mm, flat footed UIC $60-25$ ton axle load, pre-stressed concrete

$1 \quad$ Railroad track sleeper.

Max $1.5 \%$ max grade (loaded \& unloaded), $800 \mathrm{~m}$ min curve radius.

$2 \quad$ Operating

320 days/annum, 21 hour average turn around/trip.

Speed: $80 \mathrm{~km} /$ hour (straight), $40 \mathrm{~km} /$ hour (curve).

3 locations: Tabapenanjung, Kota Padang and MuaraEnim

3 Train loading station

Facilities: balloon loop, crushing, stockyard, train loader, office. 
4 Train set operation

5 Train

$6 \quad$ Crossing

7 Tunnel

$8 \quad$ Signaling and communication

$9 \quad$ Railroad facilities and utilities
21 train sets for 40 MTPA: 42 locomotives \& 1575 wagons.

Electric powered, with Diesel unit for emergency (4 locomotives).

Major bridges $(>300 \mathrm{~m}): 18$ units, minor bridges $(<300 \mathrm{~m}): 17$ units.

$4 \mathrm{~km}$ in length.

Computerized and distance monitoring.

Marshaling yard, maintenance stations, siding stations, switching, security fencing, drainage, loading station facilities \& utilities.

\section{MAIN EQUIPMENT}

1 Wagon dumper/ tippler

2 Stacker and re-claimer

3 Ship loader

4 Conveyor belt

$5 \quad$ Locomotive SS4B type (electric)

$6 \quad$ Locomotive SS4B type (diesel)

7 Train wagon

D POWER

$1 \quad$ Electricity demand

2 Electricity supply

\section{Coal Handling and Railroad}

O type, triplicate wagon dumper (triplet), capacity: 27 cy/hour.

Slewing 44m, counter 295 tons, height: $13.5 \mathrm{~m}$, capacity: 6,000 tons/hour.

Long traveling, luffing type, capacity: 6,000 tons/hour.

Shed type, width: $2,000 \mathrm{~mm}$, speed: $5 \mathrm{~m} /$ second.

6,400 HP, average speed: $80 \mathrm{~km} /$ hour, max speed: 100 $\mathrm{km} /$ hour.

8,700 HP, average speed: $80 \mathrm{~km} /$ hour, max speed: 100 $\mathrm{km} /$ hour.

C80 wagon, capacity: 80 ton, $\max$ speed: $100 \mathrm{~km} /$ hour.

\section{Electricity from Power Plant}

165 MW for Railroad, 30 MW for Port (200MW for whole operation).

Mine Mouth Coal Fired Steam Power Plant (developed by sister's company)

Underdevelopment State Owned Geothermal Power Plant

Source: Kurniawan, 2010; CCCC, 2008 - 2009; CCFD, 2009; CNR Datong, 2009; CRCC, 2009; CSR, 2006; Dahlian, 2009; PratamaWidya, 2008 - 201; QRRS, 2009; and Shenyang, 2009 


\section{EXPORT AND ECONOMIC BASE THEORIES}

Figure 4 shows an overview map of coal mines and resources along the railroad alignment which has been estimated at 16.6 billion tons of coal resources (Kurniawan, 2010). According to the export-base theory, the abundant coal resources may promote opportunity for economic growth in Bengkulu Province from coal exploitation, transportation and export through the Pulau Baai Port in Bengkulu.

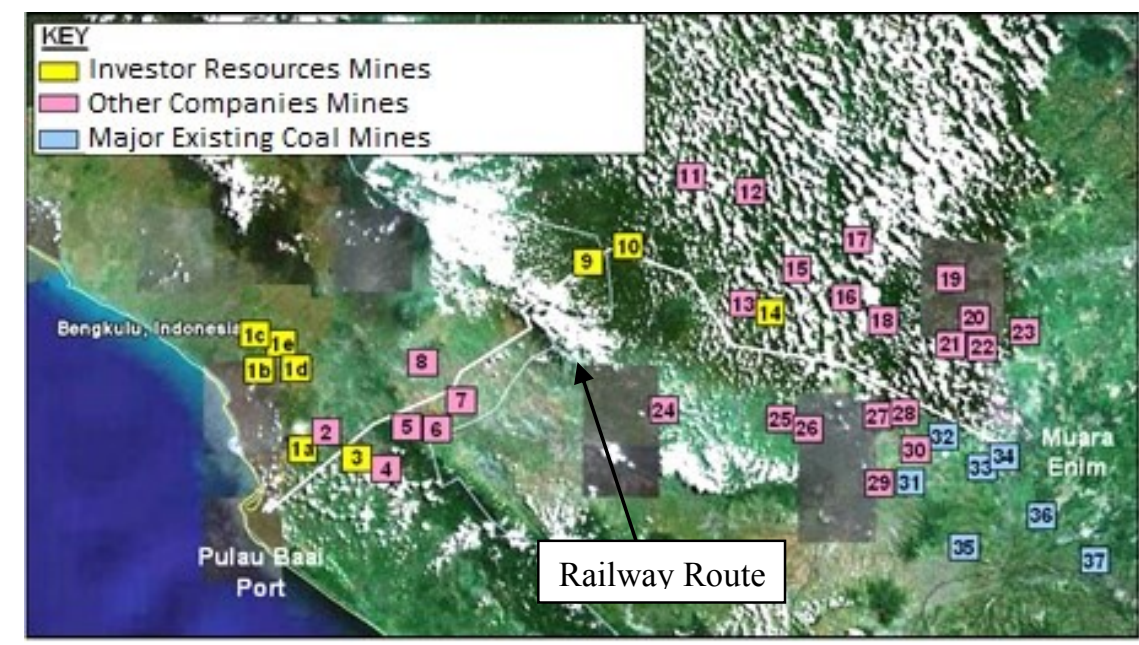

\begin{tabular}{clll}
\hline COAL & \multicolumn{1}{c}{ CALORIE } & \multicolumn{1}{c}{ RESERVES } & \multicolumn{1}{c}{ REMARKS } \\
& \multicolumn{1}{c}{ (MILLION TON) } & \\
\hline $4500-7100$ & 117.25 & Investor Resources' Mines \\
$<5100,5000-7000$ & $10,388.78$ & Other Companies' Mines \\
$<5100,5000-7000$ & $6,105.96$ & Major Existing Coal Mines \\
\hline \multicolumn{2}{c}{ Total Resources $=16,611.99$} & & \\
\hline
\end{tabular}

Source: Kurniawan, 2010

\section{Figure 4: Map of Coal Mines along the Railroad Alignment}

Coal is one of the most important commodities globally today and is one of the main fuel source for the world's electricity production. According to US Energy Information Administration the worldwide coal consumption has doubled since 1980, mainly driven by increase in Asia as shown in Figure 5. The Asian demand is dominated by China and India, where the former accounts for 73 percent of Asia's 
consumption and almost half of global coal consumption. Furthermore, the domestic demand for coal is also increasing to meet the Indonesian energy shortage.

According to the economic base theory, this promising coal demand particularly in China and India as well as from Indonesian market, and together with the introduction of this coal transportation Project, will determine significant economic growth in Bengkulu Province.

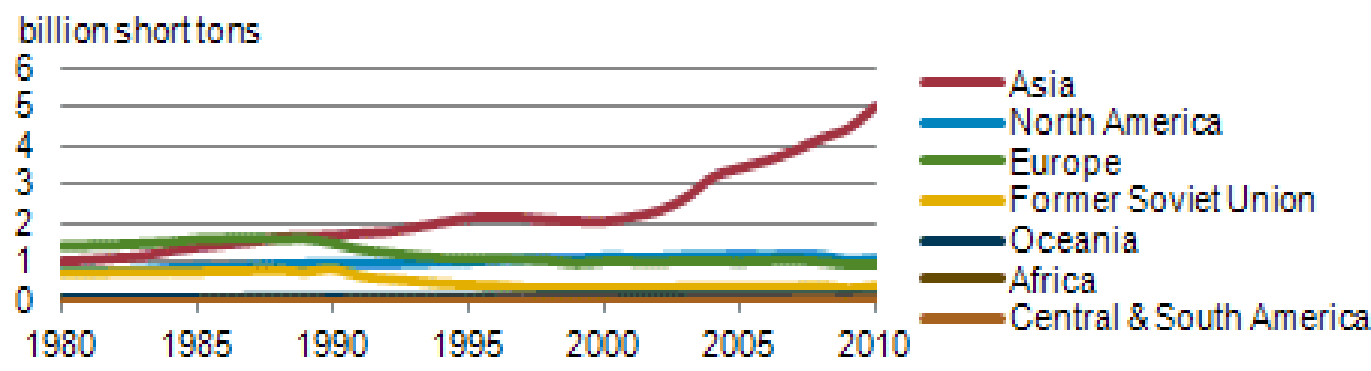

a. World Coal Consumption by Region (US Energy Information Administration)

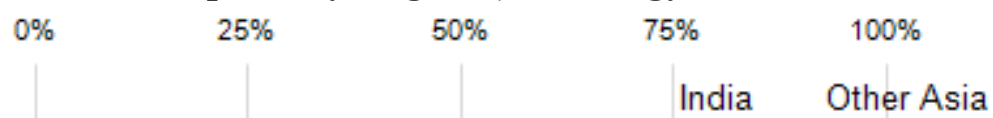

China

Japan

\section{b. Coal Consumption Share in Asia (U.S. Energy Information Administration, 2011)}

Figure 5: Worldwide Coal Consumption

\section{COST-BENEFIT ANALYSIS}

The Project investment value $=$ Project cost + contingency fee + cost of debt. The Project cost covers the entire development cost from design stages, licenses, land acquisition, construction, and procurement of main coal processing equipments until project handover. Contingency fee is the unexpected cost incurred during construction due to an inaccuracy, price escalation, and other unpredictable issues, while cost of debt is a value of interest during the construction. Table 2 shows the estimated Project investment value, and Figure 6 shows the cash flow distribution during the Project construction that is estimated at 3.5 years. 
Table 2: Investment Value of the Coal Transportation Project

\begin{tabular}{|c|c|c|}
\hline NO & ITEM & $\begin{array}{c}\text { TOTAL COST } \\
\text { (USD) }\end{array}$ \\
\hline 1 & Feasibility study, Engineering design and Environmental study & $50,655,375$ \\
\hline 2 & Dredging work (from existing up to $-15 \mathrm{~m}$ at channel $\&$ basin) & $80,006,750$ \\
\hline 3 & Coal port (for 3 Panamax vessels) including facilities & $310,548,054$ \\
\hline 4 & Stockyard area $\left(2.000 \times 500 \mathrm{~m}^{2}\right)$ including facilities & $364,667,266$ \\
\hline 5 & Double track railroad $(265 \mathrm{~km})$ including facilities & $2,077,341,627$ \\
\hline 6 & Cost of debt, interest payments during construction & $120,000,000$ \\
\hline \multirow[t]{2}{*}{7} & Contigency cost & $139,994,928$ \\
\hline & Total (excluding VAT) & $3,143,214,000$ \\
\hline
\end{tabular}

Source: Pratama Widya, 2010b

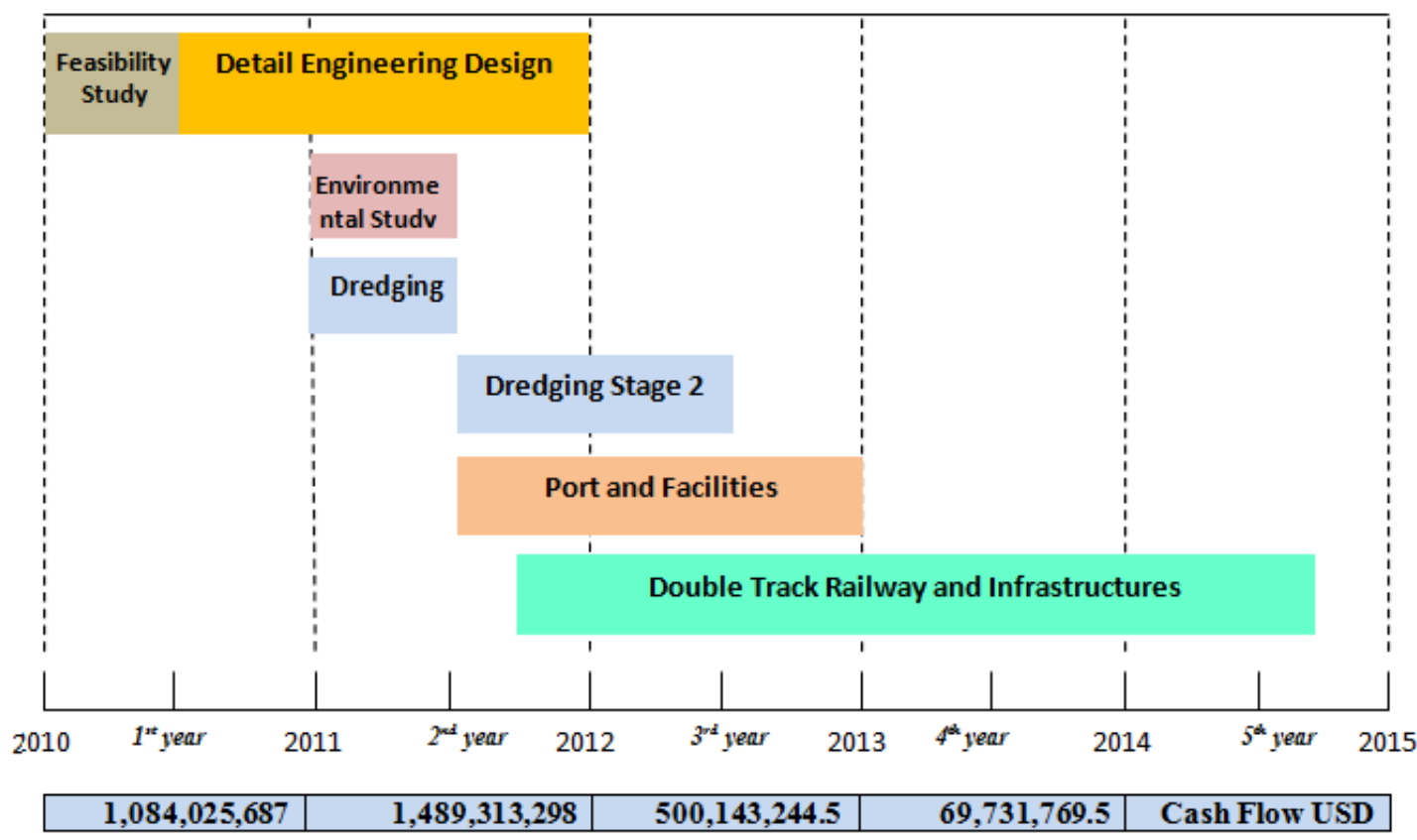

Source: Kurniawan, 2010

Figure 6: Project Time Schedule and Cash Flow during Implementation 
Based on the investment value, this Project can be categorized as a Mega Infrastructure Project that requires substantial investment funds. The financial model adopted for this study is as follows:

Investor equity : $30 \%$

Loan/ external fund: 70\% ("Joint In", "Joint Support" and "Public Support" to the potential investor partners, banks and public market)

Revenue for this Project will be mainly from unloading service fee at the developed port, vessel passing fee through the dredged Pulau Baai channel, and railroad transportation fee from the coal mines to the Pulau Baai port. Table 3 shows the Project revenue during the project lifetime. The unit rate fees are according to the investor contract agreement with the coal mine clients.

Table 3: Project Revenue Sources

\begin{tabular}{|c|c|c|c|c|}
\hline \multirow[t]{2}{*}{ No. } & \multirow[t]{2}{*}{ ITEM } & \multirow{2}{*}{$\begin{array}{l}\text { VOLUME } \\
\text { Million mT }\end{array}$} & \multirow{2}{*}{$\begin{array}{l}\text { CONTRACT } \\
\text { RATE } \\
\text { USD / mT }\end{array}$} & \multirow{2}{*}{$\begin{array}{c}\text { YEARLY } \\
\text { REVENUE } \\
\text { Million USD }\end{array}$} \\
\hline & & & & \\
\hline A & $\underline{\text { Port }}$ & & & \\
\hline & Unloading fee at Pulau & & & \\
\hline A. 1 & Baai Port, Bengkulu & 40 & 5 & 200 \\
\hline & Channel fee at & & & \\
\hline A. 2 & Pulau Baai, Bengkulu & 40 & 4 & 160 \\
\hline B & $\underline{\text { Railroad }}$ & & & \\
\hline B. 1 & $\begin{array}{l}\text { Coal mines at } \\
\text { intermediate hinterland of } \\
\text { railroad route, }\end{array}$ & 20 & 14.5 & 290 \\
\hline B. 2 & $\begin{array}{l}\text { Major existing coal mines in } \\
\text { Muara Enim }\end{array}$ & 20 & 14.5 & 290 \\
\hline & Total & & & 850 \\
\hline
\end{tabular}

Note: Contract rate will increase in every 8 years after operation starts. 
Table 4: Assumed Project Financial Analysis

\begin{tabular}{|c|c|c|}
\hline No & ITEM & REMARKS \\
\hline 1 & Inflation Rate & $\begin{array}{l}\text { Year 2010: } 6.96 \% \text { (Bank of Indonesia). } \\
\text { Assumed rate: Year } 1-5: 6 \% \text {, Year } 6-10: 5 \% \text {, Year } 11-30 \text { : } \\
4 \% \text {. }\end{array}$ \\
\hline 2 & Operation and Maintenance & $\begin{array}{l}\text { Basic operation: } 1 \% / \mathrm{yr} \text { of investment } \\
\text { Basic maintenance: } 1.5 \% / \mathrm{yr} \text { of investment } \\
\text { Electricity: US } \$ 0.8 / \mathrm{kwh} \text { (under development state-owned } \\
\text { geothermal power plant). }\end{array}$ \\
\hline 3 & $\begin{array}{l}\text { Miscellaneous Expenses } \\
\text { and Salvage Value }\end{array}$ & $\begin{array}{l}\text { Miscellaneous expenses and royalty: } 0.5 \% / \mathrm{yr} \text { of operation cost. } \\
\text { Salvage value: } 1 \% \text { of investment. }\end{array}$ \\
\hline 4 & Settlement and Interest & $\begin{array}{l}\text { Settlement period: } 15 \text { years (after construction completed). } \\
\text { Loan model: } 70 \% \text { of investment (USD 2,200,249,800). } \\
\text { Interest rate: } 10 \% \text { of flat (Bank of Indonesia, 2010). }\end{array}$ \\
\hline 5 & Depreciation & Basic depreciation period: 15 years. \\
\hline 6 & Tax & Tax value: $30 \%$ of net income (Indonesian Tax Regulation). \\
\hline
\end{tabular}

Source: Kurniawan, 2010

The cost-benefit analysis of the Project is based on the Project revenue and cost drivers (Tables 3 and 4). The financial analysis for the first stage of Project lifetime (30 years of operation) is summarized in Table 5, where three analysis models are adopted: (1) Cost- Benefit Ratio; (2) Internal Rate of Return (IRR); and (3) Payback Period. 
Table 5: Cost-Benefit Analysis during the Project Lifetime

Table 5.1: Benefit-Cost Ratio and Internal Rate of Return

\begin{tabular}{|c|c|c|c|c|c|c|c|c|c|c|c|c|c|c|c|c|}
\hline \multicolumn{2}{|r|}{ YEAR OF } & \multirow{2}{*}{\begin{tabular}{c|} 
INVESTMENT \\
(USD)
\end{tabular}} & \multirow{2}{*}{$\begin{array}{l}\text { OPERATION AND } \\
\text { MAINTENANCE } \\
\text { (USD) }\end{array}$} & \multirow{2}{*}{\begin{tabular}{|l|} 
MISCELLANEOUS \\
EXPENSES (USD)
\end{tabular}} & \multirow{2}{*}{\begin{tabular}{|c|} 
SEITLEMENT \\
(USD)
\end{tabular}} & \multirow{2}{*}{$\begin{array}{l}\text { INTEREST } \\
\text { (USD) }\end{array}$} & \multirow{2}{*}{$\begin{array}{c}\text { DEPRECIATION } \\
\text { (USD) }\end{array}$} & \multirow{2}{*}{\begin{tabular}{c|} 
REVENUEI \\
BENEFTI (USD)
\end{tabular}} & \multirow{2}{*}{$\begin{array}{c}\text { NET INCOME } \\
\text { (USD) }\end{array}$} & \multirow{2}{*}{$\operatorname{TAX}$ (USD) } & \multirow{2}{*}{$\begin{array}{c}\text { CASH FLOW } \\
\text { (USD) }\end{array}$} & \multicolumn{2}{|c|}{ PRESENT VALUE (USD) } & \multirow{2}{*}{$\begin{array}{l}\left.\mathrm{NPV}_{0}\right) \\
\left(\mathrm{NPP}_{0}-\right. \\
\left.\mathrm{NPV}_{2}\right)^{-}\end{array}$} & \multirow{2}{*}{\begin{tabular}{|c|} 
COST \\
BENEFIT \\
RATIO
\end{tabular}} & \multirow[b]{2}{*}{ IRR (\%) } \\
\hline No & PROJECT & & & & & & & & & & & $10 \%$ & $15 \%$ & & & \\
\hline 1 & 2011 & 751.229 .801 & 7568.180 & 37.941 & & & & - & $\frac{.626 .121)}{(7.626}$ & - & $\frac{17.626 .121)}{(1)}$ & $|(6.932 .144)|$ & (6.630.912) & & & \\
\hline 2 & 2012 & 1.032 .094 .116 & 10.425 .193 & 52.126 & & & & - & $\mid \begin{array}{l}\mid 10.477 .319) \\
\end{array}$ & - & $(10.477 .319)$ & $(8.658 .456)$ & (7.921.901) & & & \\
\hline 3 & 2013 & 346.599 .268 & $\begin{array}{ll}3.501 .003 \\
\end{array}$ & 17.505 & & & & - & $(3.518 .508)$ & - & (3.518.508) & (2.643.455) & $(2313.419)$ & & & \\
\hline 4 & 2014 & 48.324 .116 & \begin{tabular}{|l|l|}
488.122 \\
\end{tabular} & 2.441 & & & & & $\begin{array}{l}(490.563) \\
\end{array}$ & & $(490.563)$ & $(335.055)$ & (280.455) & & & \\
\hline 5 & 2015 & & 194.808 .000 & $\begin{array}{l}974.040 \\
\end{array}$ & 146.683 .320 & 220.024 .980 & 209.547 .600 & 850.000 .000 & 77.962 .060 & \begin{tabular}{|l|l|}
23.388 .618 \\
\end{tabular} & 264.121 .042 & 163.992 .755 & 131.294 .570 & & & \\
\hline \begin{tabular}{l|l}
6 \\
\end{tabular} & 2016 & & 204.548 .400 & 1.022 .742 & 146.663 .320 & 198.022 .482 & 209.547 .600 & 850.0000000 & 90.175 .456 & 27.052 .637 & 272.670 .419 & 153.895 .185 & 117.875 .422 & & & \\
\hline 7 & 2017 & & 214.775 .820 & 1.073 .879 & 146.683 .320 & 178.220 .234 & 209.547 .600 & 850.000 .000 & 99.699 .147 & 29.909 .744 & 279.337 .003 & 143.327 .816 & 105.002 .779 & & & \\
\hline 8 & 2018 & & 225.514 .611 & $\begin{array}{l}1.127 .573 \\
\end{array}$ & 146.683 .320 & 160.398 .210 & 2095477.600 & 850.000 .000 & \begin{tabular}{|c|}
106.728 .686 \\
\end{tabular} & 32.018 .606 & 284.257 .680 & 132.606 .208 & 92.923 .836 & & & \\
\hline 9 & 2019 & & 236.790 .342 & 1.183 .952 & 146.683 .320 & 144.358 .389 & 209.547 .600 & 850.000 .000 & 1111.436 .397 & 33.430 .919 & 287.553 .078 & 121.922 .505 & 81.722 .585 & & & \\
\hline 10 & 2020 & & 248.629 .859 & 1.243 .149 & 146.683 .320 & 129.922 .550 & 209.547 .600 & 850.000 .000 & 1133.973 .522 & 34.192 .056 & 289.329 .065 & 111.536 .355 & 71.493 .212 & & & \\
\hline 11 & 2021 & & 258.575 .053 & 1.292 .875 & 146.683 .320 & 116.930 .295 & 209.547 .600 & 850.000 .000 & 116.970 .856 & \begin{tabular}{|l|}
35.091 .257 \\
\end{tabular} & 291.427 .199 & 102.116 .091 & 62.627 .705 & & & \\
\hline 12 & 2022 & & 2608.918 .055 & 1.344 .590 & 146.683 .320 & 105.237 .266 & 209.547 .600 & 850.000 .000 & 118.269 .169 & 35.480 .751 & 292.336 .018 & $\begin{array}{l}3.138 .255 \\
\end{array}$ & 54.637 .602 & & & \\
\hline 13 & 2023 & & 279.674 .777 & 1.398 .374 & 146.683 .320 & 94.713 .539 & 209.547 .600 & 850.000 .000 & 117.962 .390 & \begin{tabular}{|l|l|}
35.394 .717 \\
\end{tabular} & 292.135 .273 & 84.602 .375 & 47.471 .982 & & & \\
\hline 14 & 2024 & & 290.861 .768 & 1.454 .309 & 146.683 .320 & $\begin{array}{l}85.242 .185 \\
\end{array}$ & 209.547 .600 & 986.000 .000 & 252.210 .817 & 75.663 .245 & 386.095 .172 & 101.658 .859 & 54.555 .248 & & & \\
\hline 15 & 2025 & & 302496.239 & 1.512 .481 & 146.683 .320 & \begin{tabular}{|l|l|}
76.717 .967 \\
\end{tabular} & 209.547 .600 & 966.000 .000 & 249.042 .393 & 74.712 .718 & 383.877 .275 & 91.861 .832 & 47.140 .129 & & & \\
\hline 16 & 2026 & & 314.596 .089 & 1.572 .980 & 146.683 .320 & 69.046 .170 & 209.547 .600 & 966.000 .000 & 244.553 .841 & 73.366 .152 & 380.735289 & 82.847 .999 & 40.662 .529 & & & \\
\hline 17 & 2027 & & 327.179 .932 & 1.635 .900 & 146.683 .320 & 62.141 .553 & 209.547 .600 & 986.000 .000 & 238.811 .695 & 71.643 .508 & 376.715 .786 & 74.514 .383 & 34.996 .897 & & & \\
\hline 18 & 2028 & & 340.267 .130 & 1.701 .336 & 146.683 .320 & 55.927 .398 & $209.547,600$ & 960.000 .000 & 231.873 .217 & 69.561 .965 & 371.858 .852 & 66.860 .222 & 30.046 .195 & & & \\
\hline 19 & 2029 & & 353.877 .815 & 1.769 .389 & 146.683 .320 & 50.334 .658 & 209.547 .600 & 966.000 .000 & 223.787 .218 & 67.136 .165 & 366.198 .653 & 59.873 .480 & 25.707 .145 & & & \\
\hline & 2030 & & 368.032 .927 & 1.840 .165 & & & & 966.000 .000 & 616.126 .908 & \begin{tabular}{|l|l|l|l|l}
184.838 .072 \\
\end{tabular} & 431.288 .836 & 64.069 .521 & 26.351 .748 & & & \\
\hline 21 & 2031 & & 382.754 .244 & 1.913 .771 & & & & 966.000 .000 & 601.331.984 & 180.399 .595 & 420.932 .389 & 56.867 .966 & 22.351 .510 & & & \\
\hline & 2032 & & 398.064 .414 & 1.990 .322 & & & & 986.000 .000 & 505.945 .264 & 175.783.579 & 410.161 .685 & 50.367 .855 & 18.949 .470 & & & \\
\hline 23 & 2033 & & 413.986 .991 & 2.069 .935 & & & & 1.184 .000 .000 & 767.943 .074 & 230.382 .922 & 537.560 .152 & 59.991 .713 & 2203.997 & & & \\
\hline 24 & 2034 & & 430.546 .470 & 2.152 .732 & & & & 1.184 .000 .000 & 751.300 .797 & 225.390 .239 & 525.910 .558 & 53.379 .922 & 18.354.278 & & & \\
\hline 25 & 2035 & & 447.768 .329 & 2.238 .842 & & & & 1.184 .000 .000 & 733.992 .829 & 220.197 .849 & 513.794 .980 & 47.371 .897 & 15.567 .988 & & & \\
\hline 26 & 2036 & & 465.679 .062 & 2.328 .395 & & & & 1.184 .000 .000 & 715.992 .542 & 214.797 .763 & 501.194 .780 & 42.050 .242 & 13.231 .542 & & & \\
\hline & 2037 & & 484.306 .225 & 2.421 .531 & & & & 1.184 .000 .000 & 697.272 .244 & 209.181 .673 & 488.090 .571 & 37.192 .501 & 11.177 .274 & & & \\
\hline 28 & 2038 & & 503.678.474 & \begin{tabular}{l|l}
2.52518 .392 \\
\end{tabular} & & & & 1.184 .000 .000 & 677.803.134 & 203.340 .940 & 474.462 .194 & 32.880 .230 & 9.441 .798 & & & \\
\hline 29 & 2039 & & 523.825 .613 & 2.619 .128 & & & & 1.184 .000 .000 & 657.555 .259 & 197.266 .578 & 460.288 .681 & 28.998 .187 & 7.962 .994 & & & \\
\hline \multirow[t]{3}{*}{30} & 2040 & & 544.778.637 & $\begin{array}{l}2.2723 .893 \\
2.7293\end{array}$ & & & & 1.215 .432 .140 & 667.929 .610 & 200.378 .883 & 467.550 .727 & 26.790 .657 & 7.060 .016 & & & \\
\hline & & & & & & & 3.143 .214 .000 & & \begin{tabular}{|l|l|}
9.844 .557 .999 \\
\end{tabular} & 2.960 .001 .153 & & 2.066 .165 .899 & \begin{tabular}{|l|l|}
1.133 .663 .764 \\
\end{tabular} & 2,216 & 1,608 & 21,079 \\
\hline & & & $\begin{array}{l}\text { INCLUDING 4-6\% } \\
\text { INFLATION RATE }\end{array}$ & FLAT & & & \begin{tabular}{|c|} 
DEPRECIATION \\
$10 \%$ AFTER \\
OPERATION
\end{tabular} & $\begin{array}{l}\text { FLAT REVENUE } \\
\text { EVERY 9 YEARS } \\
\text { AND WLL BE } \\
\text { ADDED BY SV AT } \\
\text { YEAR 31TH }\end{array}$ & & $\begin{array}{l}30 \% \text { FROM NET } \\
\text { INCOME AFTER } \\
\text { OPERATION }\end{array}$ & & $\begin{array}{c}\text { DF }= \\
\text { DISCOUNT } \\
\text { FACCOR AT } \\
10 \%\end{array}$ & $\begin{array}{c}\mathrm{DF}_{1}= \\
\text { DISCOUNT } \\
\text { FACTOR AT } \\
15 \%\end{array}$ & & & \\
\hline
\end{tabular}

Source: Kurniawan, 2010

Table 5.2: Payback Period

\begin{tabular}{|c|c|c|c|c|c|c|c|c|c|c|}
\hline \multicolumn{2}{|c|}{ YEAR OF } & \multirow{2}{*}{$\begin{array}{l}\text { INVESTMENT } \\
\text { (USD) }\end{array}$} & \multirow{2}{*}{$\begin{array}{c}\text { OPERATION AND } \\
\text { MAINTENANCE (USD) }\end{array}$} & \multirow{2}{*}{$\begin{array}{l}\text { MISCELLANEOUS } \\
\text { EXPENSES (USD) }\end{array}$} & \multirow{2}{*}{$\begin{array}{l}\text { DEPRECIATION } \\
\text { (USD) }\end{array}$} & \multirow{2}{*}{\begin{tabular}{|c|} 
REVENUEl \\
BENEFIT (USD)
\end{tabular}} & \multirow{2}{*}{$\begin{array}{l}\text { NET INCOME } \\
\text { (USD) }\end{array}$} & \multirow{2}{*}{ TAX (USD) } & \multirow{2}{*}{$\begin{array}{l}\text { CASH FLOW } \\
\quad \text { (USD) }\end{array}$} & \multirow{2}{*}{$\begin{array}{l}\text { CAPITAL'S } \\
\text { RETURN }\end{array}$} \\
\hline No & PROIFCT & & & & & & & & & \\
\hline & & 1 & 2 & 3 & 4 & 5 & 6 & 7 & 8 & 9 \\
\hline 1 & 2011 & 758.817 .981 & 7.588 .180 & 37.941 & & & $\begin{array}{l}(7.626 .121) \\
\end{array}$ & - & $\begin{array}{l}(7.626 .121) \\
\end{array}$ & 2.207 .875 .921 \\
\hline 2 & 2012 & 1.042 .519 .309 & 10.425 .193 & 52.126 & & & (10.477.319) & - & (10.477.319) & 2.218 .353 .240 \\
\hline 3 & 2013 & 350.100 .271 & 3.501 .003 & 17.505 & & & $(3.518 .508)$ & - & $(3.518 .508)$ & 2.221 .871 .747 \\
\hline 4 & 2014 & 48.812 .239 & 488.122 & 2.441 & & & $(490.563)$ & - & $(490.563)$ & 2.222 .362 .310 \\
\hline 5 & 2015 & & 194.808 .000 & 974.040 & 209.547 .600 & 850.000 .000 & 444.670 .360 & 133.401 .108 & 520.816 .852 & 1.701 .545 .458 \\
\hline 6 & 2016 & & 204.548 .400 & 1.022 .742 & 209.547 .600 & 850.000 .000 & \begin{tabular}{|l|}
434.881 .258 \\
\end{tabular} & 130.464 .377 & 513.964 .481 & 1.187 .580 .978 \\
\hline 7 & 2017 & & 214.775 .820 & 1.073 .879 & 209.547 .600 & 850.000 .000 & 424.602 .701 & 127.380 .810 & 506.769 .491 & 680.811 .487 \\
\hline 8 & 2018 & & 225.514 .611 & 1.127 .573 & 209.547 .600 & 850.000 .000 & 413.810 .216 & 124.143 .065 & 499.214 .751 & 181.596 .736 \\
\hline 9 & 2019 & & 236.790 .342 & 1.183 .952 & 209.547 .600 & 850.000 .000 & 402.478 .107 & 120.743 .432 & 491.282 .275 & $(309.685 .539)$ \\
\hline
\end{tabular}

Source: Kurniawan, 2010

The cost-benefit analysis result shows the key financial values of the Project: Costbenefit ratio of 1.61 , IRR of $21.1 \%$, and payback period of 5 years.

The Project cost-benefit ratio is much greater than 1.0, indicating that the Project produces benefits from the investment, and according to Gray (1986), is considered feasible.

The Project IRR of $21.1 \%$ being greater than the adopted interest rate or discounted market rate of 10\% (Bank of Indonesia, 2010) indicates a high rate return from the investment. Although the interest rate is assumed flat for the 30-year period of Project lifetime, the high IRR value may still accommodate the possibility of increasing 
interest rate. It is however unlikely that the interest rate may be higher than $15 \%$, and the current trend shows reductions in interest rate (Bank of Indonesia, 2010). According to the general practice in oil and gas project in Indonesia, the acceptable minimum IRR value is $15 \%$ (Sinaga, 2009) due to the high risk degrees associated in the energy sector. As considered a Mega Project with high risks, the Project IRR value also meets the above-suggested IRR value. According to Sinaga (2009), a Project with payback period of 5 years is considered worth investing.

The cost-benefit analysis therefore suggests that this Project is financially feasible to develop and operate for a 30-year period of project lifetime, and presented high financial values are attractive in the eyes of investors.

\section{IMPACT TO BENGKULU GROSS DOMESTIC PRODUCT}

Regression and path analyses based on secondary data produce the following summary of direct, indirect and total influences between variables presented by the feasibility study:

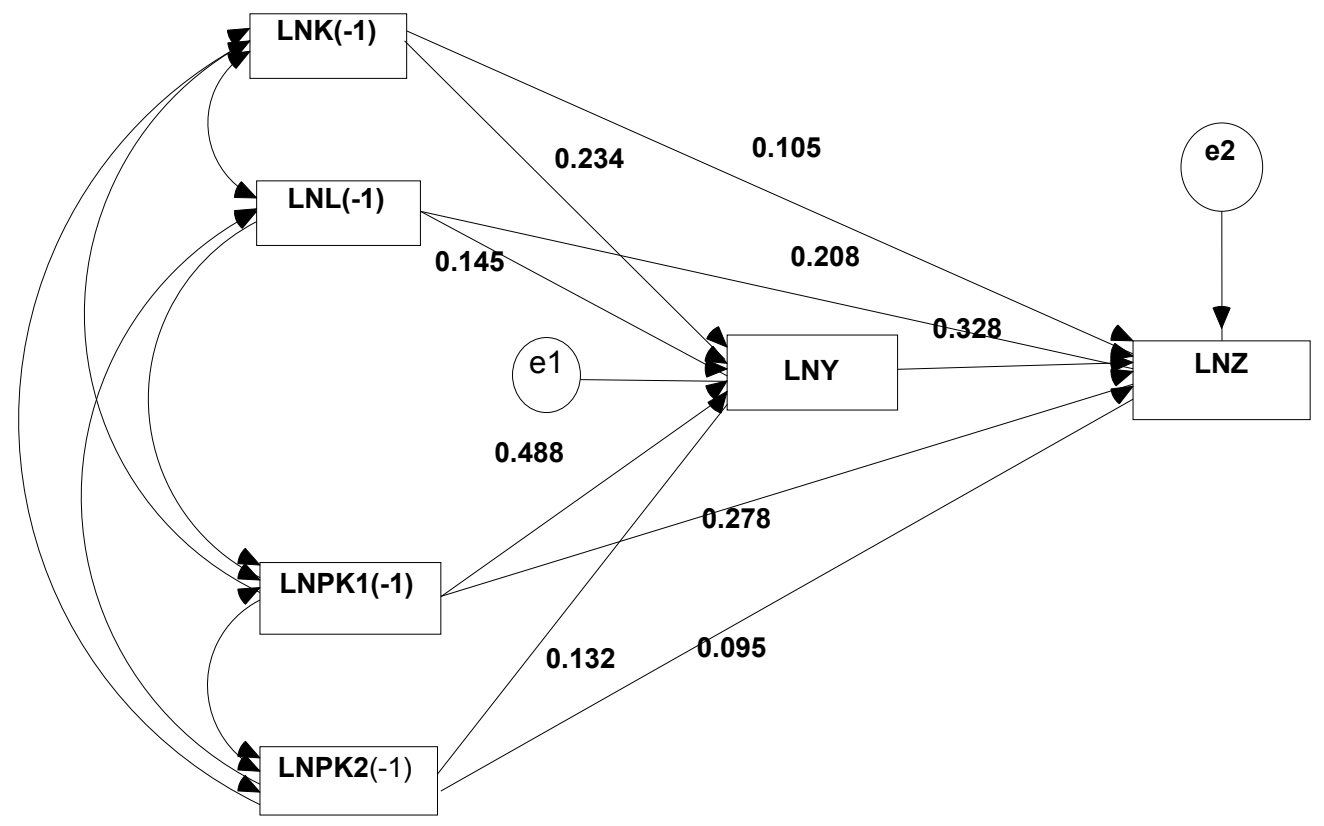

Figure 7: Framework of Direct, Indirect and Total Influences between Variables 
Table 6: Summary of Direct, Indirect and Total Influences between Variables

\begin{tabular}{|c|c|c|c|}
\hline \multirow{2}{*}{$\begin{array}{c}\text { Independent } \\
\text { Variable }\end{array}$} & \multirow{2}{*}{ Influence Type } & \multicolumn{2}{|c|}{ Dependent Variable } \\
\hline & & LNY & LNZ \\
\hline & Direct Influence & 0.234 & 0.105 \\
\hline \multirow[t]{3}{*}{$\operatorname{LNK}(-1)$} & Indirect Influence & - & 0.077 \\
\hline & Total Influence & 0.234 & 0.182 \\
\hline & Direct Influence & 0.145 & 0.208 \\
\hline \multirow[t]{3}{*}{$\operatorname{LNL}(-1)$} & Indirect Influence & - & 0.048 \\
\hline & Total Influence & 0.145 & 0.256 \\
\hline & Direct Influence & 0.488 & 0.278 \\
\hline \multirow[t]{3}{*}{ LNPK1(-1) } & Indirect Influence & - & 0.160 \\
\hline & Total Influence & 0.488 & 0.438 \\
\hline & Direct Influence & 0.132 & 0.095 \\
\hline \multirow[t]{3}{*}{ LNPK2(-1) } & Indirect Influence & - & 0.043 \\
\hline & Total Influence & 0.132 & 0.138 \\
\hline & Direct Influence & - & 0.328 \\
\hline \multirow[t]{2}{*}{ LNY } & Indirect Influence & - & - \\
\hline & Total Influence & - & 0.328 \\
\hline
\end{tabular}

Source: Kurniawan, 2012

The analysis data shows that private output has significant contributions to increase in GDP of Bengkulu Province.

One of major contributions from the Project to Bengkulu GDP is its corporate income tax paid to the Bengkulu Local Government (PEMDA). Bengkulu and National Income Tax Agency in 2011 received a contribution of $3.4 \%$ and $70 \%$ from their GDP respectively (Indonesian Director General of Taxes). The Project income tax is set at $30 \%$ according to Indonesian Tax Regulation as shown in Figure 8 


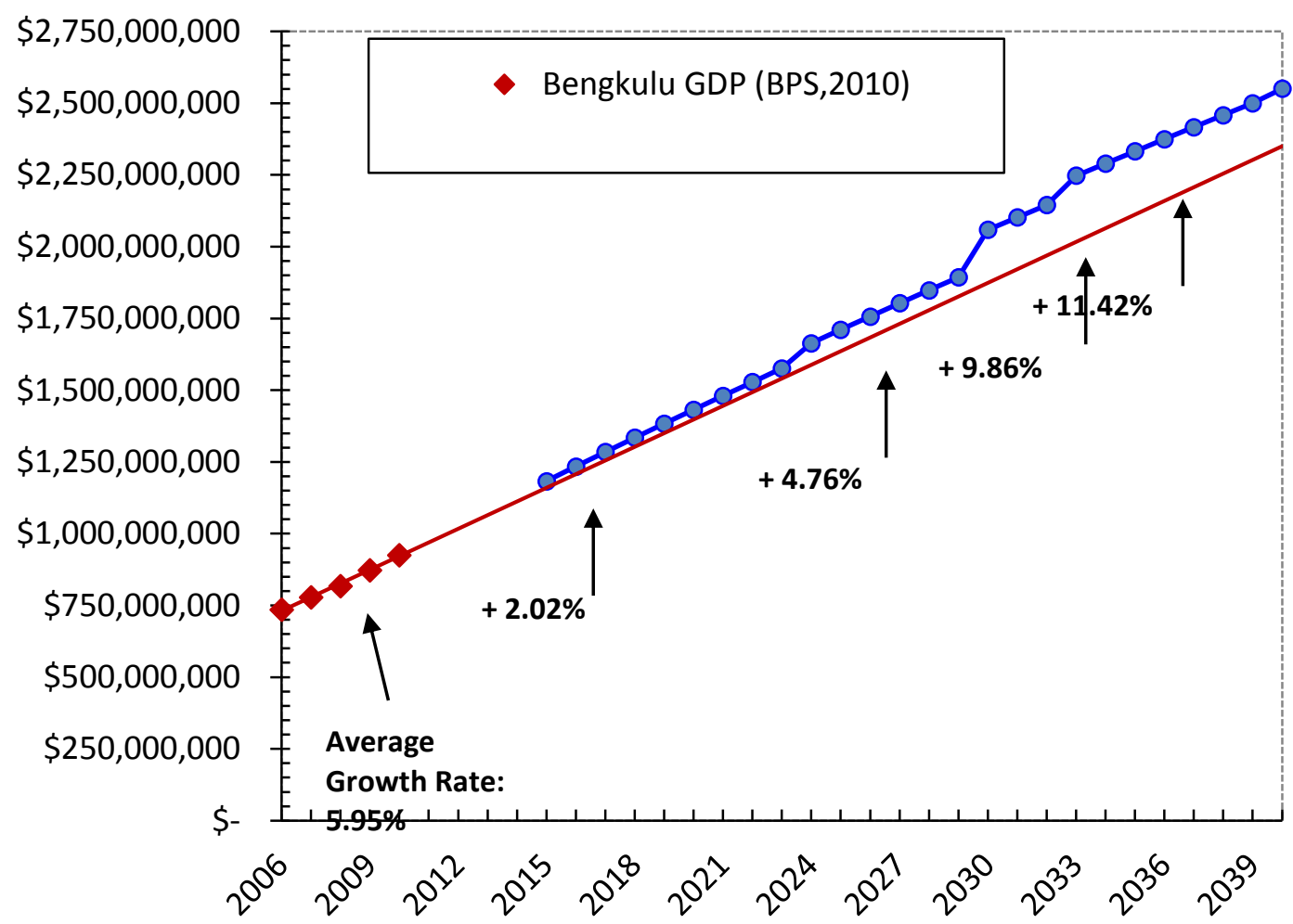

Figure 8: Impact of Project Income Tax on Bengkulu GDP Growth

Figure 8 presents the impact of Project income tax on the growth of Bengkulu GDP during the Project lifetime. The Bengkulu GDP growth is optimistically predicted to be linear based on the statistic data of Bengkulu GDP with a constant price base (BPS Provinsi Bengkulu, 2010) and compared with the addition of Project income tax.

It shows that after the Project comes into operation, the Project income tax will increase the Bengkulu GDP by $2.02 \%$ in 2015 . The Bengkulu GDP growth will rise higher, by $4.76 \%$ by 2024 , due to increases in the Project revenue (coal transportation unit rate increases in every eight years), and $9.86 \%$ by 2030 due to diminishing cost items such as settlement, interest payment and depreciation. With one unit increase in coal transportation unit rate, the Bengkulu GDP growth is estimated at $11.42 \%$ by 2033. This income tax contribution especially after 2024 gains a growth rate equal to, or higher than, the average GDP growth rate from year 2006 to 2010 (5.95\%). 
Furthermore, it is estimated that the Project income tax may only account for $1.98 \%$ of Bengkulu GDP at the beginning, but it will be a significant income for Bengkulu GDP by 2033, providing $10.25 \%$ of the total Bengkulu GDP as shown in Figure 9.

The above discussions indicate that the Project income tax alone provides significant benefits for Bengkulu GDP growth. This therefore highlights the importance of the Project to the economy of Bengkulu Province.

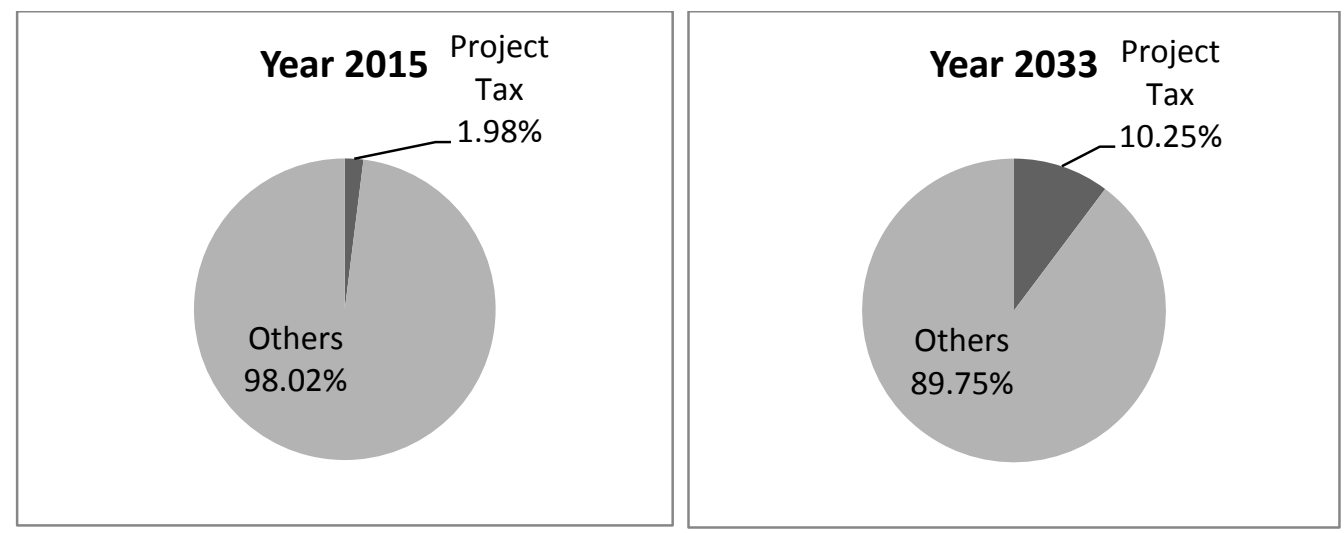

Figure 9: Share of Project Income Tax in Bengkulu GDP

Further studies can be conducted to analyze the impact of this Project operations to other economic indicators, such as: income per capita, local and regional developments, employment opportunities, infrastructure development, and export of other commodities through Pulau Baai Port, Bengkulu.

\section{IMPACT ON BENGKULU UNEMPLOYMENT}

Working population in Indonesia consist of people of age 15 to 64 . In the last four years, the number of laborers in Bengkulu has increased significantly. The working population in Bengkulu in 2012 comprised 1,769,053 people, with an increase of $7.56 \%$ in the years 2005- 2012, (BPS, 2012).

The quality of labor force in Bengkulu in terms of education is considered relatively low. Most Bengkulu workers only finish primary education.

Bengkulu labor force by education and employment in May 2012 can be seen in Table 7. 
Table 7: Labor Force in Terms of Education and Employment in May 2012 ( BPS 2012)

\begin{tabular}{lllll}
\hline No. & \multicolumn{1}{c}{ Labor quality } & As \% & \multicolumn{1}{c}{ Labor quality } & As \% \\
\hline A & Education Level & & Employment & \\
1 & Non educated / primary school drop & & & 58.53 \\
& out and primary school graduated & 47.22 & Plantation & 15.31 \\
2 & Junior high school graduated & 21.72 & Trading & 14.12 \\
3 & Senior high school graduated & 22.89 & Services & 12.04 \\
\hline
\end{tabular}

Source: BPS 2012

Labor force participation rate (TPAK) is a ratio of the labor force and the overall size of national population of the same age range in 2012 , namely $74.85 \%$. Therefore, Bengkulu has a total of $74.85 \%$ x 1,220,800 or $913,768.8$ workers.

The followings are estimated demand for labor for the Project:

Table 8: Estimated Labor Demand for the Construction of Coal Port and Railroad Project Without Their Multiplier Effects

\begin{tabular}{llccc}
\hline No. & \multicolumn{1}{c}{ Item } & \multicolumn{3}{c}{ Labor demand } \\
\cline { 3 - 5 } & & $\begin{array}{c}\text { Expatriate } \\
\text { (Men/ } \\
\text { Month) }\end{array}$ & $\begin{array}{c}\text { Skilled } \\
\text { (Men/ } \\
\text { Month) }\end{array}$ & $\begin{array}{c}\text { Unskilled } \\
\text { (Men/Days) }\end{array}$ \\
\hline A & Feasibility study and Engineering design & 3,043 & 39,734 & 746,633 \\
B & $\begin{array}{l}\text { Dredging work (From existing up to - 15m at } \\
\text { channel \& basin) }\end{array}$ & 7,073 & 9,079 & $4,340,079$ \\
C & Coal port (For 3 Panamax sizing) & 3,300 & 57,750 & $24,458,675$ \\
D $\quad$ Stockyard area (2,000 X 500 M2) & 1,995 & 12,470 & $8,009,917$ \\
E & $\begin{array}{l}\text { Double-track railroads (265 Km) and 20-Km } \\
\text { balloon loop }\end{array}$ & 6,200 & 31,719 & $13,047,958$ \\
& Total (Person) & & & \\
& Total demand (Person) & 515 & 3,589 & 39,611 \\
& & 43,715 & & \\
\hline
\end{tabular}


Table 9: Estimated Labor Demand for Operation of Coal Port and Railroad

\begin{tabular}{lccc}
\hline \multirow{2}{*}{ Operational Activities } & \multicolumn{2}{c}{ Estimated Labor Demand (Person) } \\
\cline { 2 - 4 } & Expatriate & Skilled & Unskilled \\
\hline Jakarta Management Head Office & 6 & 22 & 10 \\
Coal Port Operation & 31 & 205 & 237 \\
Coal Railroad Operation & 23 & 279 & 279 \\
Maintenance Dredging & 2 & 15 & 25 \\
Total & & $\mathbf{1 , 1 3 4}$ & \\
\hline
\end{tabular}

Ministry of Indonesian Public Works, in 2007, conducted a research on contribution from infrastructure projects to job opportunities within projects themselves and in other sectors as well. The result shows that a project worth one trillion rupiah may create employment for 28,000 workers. Therefore, this Project capitalized at US $\$ 3,143,124,000$ may create $31 \times 28,000=868,000$ jobs.

The statistical analysis from feasibility study shows that 30 time series data indicate $24,648,000$ workers totally can be absorbed during 30 years with an average of 821,600 workers every year with standard deviation of 144,450. Employment trend graphs show the labor force tends to increase from year to year (Table 10 and Figure 10).

Table 10: Labor Description Data

Statistics

L: Labor (ribu orang)

\begin{tabular}{|ll|l|}
\hline N & \multicolumn{1}{|c|}{ Valid } & 30 \\
& Missing & 0 \\
Mean & & 821.60 \\
Std. Deviation & 144.45 \\
Minimum & 168.00 \\
Maximum & 882.00 \\
Sum & $24,648.13$ \\
\hline
\end{tabular}

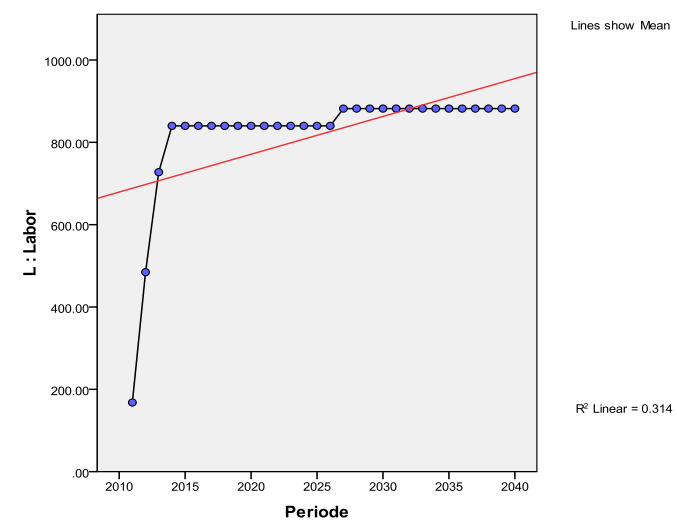

Figure 10: Labor Trend 


\section{CONCLUSION}

This paper analyzes the impact of the railroads for coal transportation from Muara Enim, Sumatra Selatan Province, to the new port at Pulau Baai, Bengkulu, on Bengkulu GDP. The study combines the theories of export and import base, economic and regional developments, economic impact study and cost-benefit analysis with the empirical data. The results show that the Project is financially feasible and has high attractiveness towards investors. The generated Project tax income from output promotes the Bengkulu GDP growth, provides a major income to Bengkulu GDP and helps reduce unemployment rate. This study also shows the significant and important value of the Project to the Bengkulu economy and decreases in the unemployment.

\section{ENDNOTES}

The author wishes to express gratitude to Prof. Dr. Made Kembar Sri Budhi, Drs.MP, Prof. Dr. Made Suyana Utama, SE, MS and Dr. Nyoman Mahaendra Yasa. SE. MSi from Faculty of Economics at Postgraduate Program, University of Udayana, Denpasar, Indonesia, as his dissertation promoters and co-promoters I and II respectively, for their sincere guidance and advice for the completion of this paper. Appreciation and gratitude are also expressed to reviewers and publisher, who have been providing for publishing this paper. Nevertheless, the author feels alone remains responsible for any error and omissions left in this paper

\section{References}

Anderson, David (2011), Cracking the AP Economics Macro \& Micro Exam, The Princeton Review, Random House, Inc. New York, 2011 Edition.

Arsyad, Lincolin (2010), Pembangunan Ekonomi Unit, STIM YKPN, Yogyakarta.

BPS (Bengkulu Statistic Center) Bengkulu Province (2009), Indikator Kesejahteraan Rakyat / Welfare Indicators, BPS, Jakarta, Cataloque No: 4102004.

(2009), Provinsi Bengkulu dalam Angka, Publisher: BPS Provinsi Bengkulu, Katalog BPS: 1102001.17.

(2010), Provinsi Bengkulu dalam Angka, Publisher: BPS Provinsi Bengkulu, Katalog BPS: 1102001.17.

(2012), "Data from Library of Central Bureau of Statistics", Bengkulu Province (unpublished). 
Capozzi ,Catherine (2010), "Economic Analysis for Business", http://www.ehow.com/info_7841935_economic-analysis-business.html.

Gray, C. et al. (1986), Pengantar Evaluasi Proyek, Gramedia, Jakarta.

Hall, S. (2010a), "What Is Economic Analysis?" Department of the Army: Economic Analysis Description and Methods. http://www.ehow.com/about_5345033_research-methodologyeconomics.html.

(2010b), "Economic Impact Analysis", Economic Development Research and Training Center: Economic Impact Analysis, http://www.ehow.com/about_7285526_tools-economic-impactanalysis.html.

(2010c), "Types of Economic Evaluations", Interactive Textbook on Clinical Sympton Research: Clincial Economics - Types of Economic Evaluation, http://www.ehow.com/list_6744942_types-economic-evaluations.html.

Kurniawan, P. (2010), "Feasibility Study, Coal Port and Double Track Railway from Muara Enim to Pulau Baai Transportation Project to Increase Export Capacity in Bengkulu", under PT. Pratama Widya, Jakarta, (unpublished).

(2012), "Economic Analysis of Transportation Development of Special Railway from Muara Enim, South Sumatra to Pulau Baai Port in Bengkulu, Indonesia”, Postgraduate Program, Economic Faculty, Udayana University, Denpasar, Indonesia, (unpublished dissertation).

Ministry of Indonesia Public Works (2007), "Kajian PUSTRA Kementerian Pekerjaan Umum" (unpublished)

Pondent, C.S. (2010), Bureau of Economic Analysis: US Economic Accounts,

Pratama Widya (2008a), “Topographical Survey at Pulau Baai and its Surrounding Area Report”, (unpublished).

(2008b), "Bathimetry Survey at Pulau Baai and its Channel Report", (unpublished). (2008c), “Offshore Geotechnical Investigation at Pulau Baai Report”, (unpublished).

(2008d), "Main Equipment Technical Specifications for Bengkulu Coal Transportation Report", (unpublished).

(2008e), “Onshore Geotechnical Investigation Report at Pulau Baai and along Railway Route ( $\pm 10 \mathrm{~km})$ ", (unpublished).

(2009a), "Environmental Study at Pulau Baai and Railway Coal Route Plan Report", (unpublished).

(2009b), "Engineering Basic Design of Railway Coal along $\pm 265 \mathrm{~km}$, at Bengkulu and South Sumatra Provinces", (unpublished).

(2010a), "Engineering Detailed Design of Coal Port at Pulau Baai," (unpublished).

(2010b), "Project Cost Estimation", (unpublished). 
QRRS (2009), "Qiqihar Railway Rolling Stock Co, Ltd, Rolling Stock Factory, China”, (unpublished).

Riley, Geoff (2006), A2 Macroeconomics / International Economy: Theories of Economic Growth, http://tutor2u.net/economics/revision-notes/a2-macro-theories-of-economic-growth.html.

Shenyang (2009), "Shenyang Mining Machinery Works, Conveyor Belt Contractor and Supplier" (unpublished).

Sinaga, Dadjim (2009), Studi Kelayakan Bisnis dalam Ekonomi Global, Teori dan Aplikasinya dalam Evaluasi Proyek, Mitra Wacana Media, Jakarta.

Stimson, Robert J., R.R. Stough \& B.H. Roberts (2006), Regional Economic Development: Analysis and Planning Strategy, Revised Edition., Berlin, Springer

Stimson, Robert J., R.R. Stough, \& M. Salazar (2009), Leadership and Institutions in Regional Endogenous Development, George Mason University, VA, USA.

U.S. Energy Information Administration (2011), www.eia.gov. 